\title{
Propagation of inhomogeneous plane waves in isotropic solid crystals
}

\author{
Livia Harabagiu and Olivian Simionescu-Panait
}

\begin{abstract}
In this paper we study the impact of initial mechanical deformation and electric fields applied to linear elastic isotropic solid, on the propagation of inhomogeneous plane waves in such media. We derive the decomposition of the propagation condition for particular isotropic directional bivectors and we show that the specific coefficients are similar to the case of guided waves propagation in isotropic solids subject to a bias.
\end{abstract}

\section{Introduction}

Last decades, the problems related to electroelastic materials subject to incremental fields superposed on initial mechanical and electric fields have ganied considerable extension, due to their complexity and to multiple applications. The basic equations of piezoelectric bodies subject to infinitesimal deformations and fields, superposed on initial deformation and electric fields, were given by Eringen and Maugin in the well-known monography [9].

While the concept of bivector is described in [6], the algebra of bivectors is well established in [3], [5] and [18]. Inhomogeneous plane waves arise in many areas of continuum mechanics .

Key Words: Inhomogeneous plane waves, isotropic solids, initial electromechanical fields, isotropic directional bivectors.

2010 Mathematics Subject Classification: 74 J05, 74B15, 74E15, 74F15.

Received: December, 2014

Revised: February, 2015

Accepted: March, 2015 
Important examples using this concept may be found in [1] for anisotropic elasticity, in [2] for electromagnetism, in [4] for wave propagation in porous materials.

In this paper we investigate the conditions of inhomogeneous plane waves propagation in isotropic solids subject to initial mechanical and electric fields. We obtain the components of the electroacoustic tensor for the class 2 and class $m$ of the monoclinic system with coefficients similar to the case of guided wave propagation in isotropic solid.

\section{Basic equations. Condition of propagation}

We assume that the elastic dielectric is nonmagnetizable and conducts neither heat, nor electricity. We shall use the quasi-electrostatic approximation of the equations of balance. Furthermore, we assume that the elastic dielectric is linear and homogeneous, that the initial homogeneous deformations are infinitesimal and that the initial homogeneous electric field has small intensity. This problem may be found in the monographic chapter [12].

In this case the homogeneous field equations take the form:

$$
\begin{gathered}
\stackrel{\circ}{\mathbf{u}}=\operatorname{div} \boldsymbol{\Sigma}, \operatorname{div} \boldsymbol{\Delta}=0, \\
\operatorname{rot} \mathbf{e}=0 \Leftrightarrow \mathbf{e}=-\operatorname{grad} \varphi
\end{gathered}
$$

where $\stackrel{\rho}{\rho}$ is the mass density, $\mathbf{u}$ is the incremental displacement, $\boldsymbol{\Sigma}$ is the incremental electromechanical nominal stress tensor, $\boldsymbol{\Delta}$ is the incremental electric displacement vector, $\mathbf{e}$ is the incremental electric field and $\varphi$ is the incremental electric potential. All incremental fields involved into the above equations depend on the spatial variable $\mathbf{x}$ and on time $\mathrm{t}$.

We suppose the following incremental constitutive equations:

$$
\begin{aligned}
& \Sigma_{k l}=\stackrel{\circ}{\Omega l m n}_{k, n} u_{m}+\AA_{m k l} \varphi_{, m}, \\
& \Delta_{k}=\stackrel{\circ}{\Lambda}_{k m n} u_{n, m}+\stackrel{\circ}{\varepsilon}_{k l} e_{l}=\stackrel{\circ}{\Lambda}_{k m n} u_{n, m}-\stackrel{\circ}{\varepsilon}_{k l} \varphi_{, l} \text {. }
\end{aligned}
$$

In these equations $\stackrel{\circ}{\Omega l m n}_{k l m}$ are the components of the instantaneous elasticity tensor, $\AA_{k m n}$ are the components of the instantaneous couplig tensor and $\stackrel{\circ}{k l}_{k l}$ are the components of the instantaneous dielectric tensor. The instantaneous coefficients can be expressed in terms of the classical moduli of the material and on the initial applied fields as follows:

$$
\begin{gathered}
\stackrel{\circ}{\Omega}_{k l m n}=c_{k l m n}+\stackrel{\circ}{S}_{k n} \delta_{l m}-e_{k m n} \stackrel{\circ}{E}_{l}-e_{n k l} \stackrel{\circ}{E}_{m}-\eta_{k n} \stackrel{\circ}{E}_{l} \stackrel{\circ}{E}_{m}, \\
\stackrel{\circ}{\Lambda}_{m k l}=e_{m k l}+\eta_{m k} \stackrel{\circ}{E}_{l},
\end{gathered}
$$




$$
\stackrel{\circ}{\varepsilon}_{k l}=\delta_{k l}+\eta_{k l}
$$

where $c_{k l m n}$ are the components of the constant elasticity tensor, $e_{k m n}$ are the components of the constant piezoelectric tensor, $\stackrel{\circ}{E}_{l}$ are the components of the initial applied electric field and $\stackrel{\circ}{S}_{k n}$ are the components of the initial applied symmetric (Cauchy) stress tensor.

From the relations (3) we find the symmetry relations:

$$
\begin{aligned}
\stackrel{\circ}{\Omega}_{k l m n} & =\stackrel{\circ}{\Omega}_{n m l k}, \\
\stackrel{\circ}{\varepsilon}_{k l} & =\stackrel{\circ}{\varepsilon}_{l k} .
\end{aligned}
$$

From the previous field and constitutive equations we obtain the following fundamental system of equations:

$$
\begin{gathered}
\stackrel{\circ}{\rho} \ddot{u}_{l}=\stackrel{\circ}{\Omega}_{k l m n} u_{m, n k}+\stackrel{\circ}{M k l}_{m, m k}, \\
\stackrel{\circ}{k m n}_{k, n k}-\stackrel{\circ}{\varepsilon n}_{k n} \varphi_{, n k}=0, i=\overline{1,3} .
\end{gathered}
$$

We suppose that the incremental displacement is defined by the inhomogeneous plane wave:

$$
\mathbf{u}(\mathbf{x}, t)=A e^{i(\mathbf{S} \cdot \mathbf{x}-\omega t)}
$$

and the incremental electric potential is defined by

$$
\varphi=\Phi e^{i(\mathbf{S} \cdot \mathbf{x}-\omega t)}
$$

where $\omega$ defines the frequency of the wave, and is a real parameter.

Here $\mathbf{A}=\mathbf{A}^{+}+i \mathbf{A}^{-}$is a complex vector defining the mechanical amplitude, $\phi$ is the electric amplitude of the wave and $\mathbf{S}=\mathbf{S}^{+}+i \mathbf{S}^{-}$is a complex vector denoting the slowness bivector. We suppose that this kind of wave propagates in an unbounded domain.

The previous relations represent a train of elliptically polarized plane waves. The waves travel in the direction of the vector $\mathbf{S}^{+}$with the slowness $\left|\mathbf{S}^{+}\right|$and are attenuated in the direction of the vector $\mathbf{S}^{-}$. For any fixed position vector $x$, the displacement vector $u^{+}$describes an ellipse similar to the one found in $[7]$.

A solution of form (6) defines an "inhomogeneous plane wave" (IPW) if the vector $\mathbf{S}^{-}$is not parallel to the vector $\mathbf{S}^{+}$. The phase speed is given by $V=\left|\mathbf{S}^{+}\right|^{-1}$, while $\left|\mathbf{S}^{-}\right|$defines the attenuation coefficient. If $\mathbf{S}^{-}$is parallel to $\mathbf{S}^{+}$we have an attenuated homogeneous plane wave.

In order to solve the problem of inhomogeneous plane wave propagation in the described material, we use the directional ellipse method, due to Hayes (see [7]). The slowness bivector $\mathbf{S}$ may be written as $\mathbf{S}=N \mathbf{C}$, where the directional bivector $\mathbf{C}$ has form $\mathbf{C}=q \mathbf{m}+i \mathbf{n}$, with $\mathbf{m} \cdot \mathbf{n}=0,|\mathbf{m}|=|\mathbf{n}|=1,|q| \geq 1$. 
$N$ is called the complex scalar slowness.Because the directional bivector $\mathbf{C}$ is prescribed, the main unkown of the inhomogeneous plane wave propagation problem is the complex scalar slowness $N$.

We have:

$$
\begin{gathered}
\dot{u}=-i \omega u, \\
\ddot{u}=-\omega^{2} u \\
u_{, i}=i \omega N C_{i} u, \\
u_{, i j}=-\omega^{2} N^{2} C_{i} C_{j} u, \\
u_{l, i j}=-\omega^{2} N^{2} C_{i} C_{j} u_{l}, \\
\varphi_{, i}=-\omega N C_{i} \varphi, \\
\varphi_{, i j}=-\omega^{2} N^{2} C_{i} C_{j} \varphi, i, j, l=\overline{1,3}
\end{gathered}
$$

Inserting (8) into (5) gives:

$$
\begin{gathered}
\stackrel{\circ}{\rho} \omega^{2} u_{l}=-\omega^{2} \stackrel{\circ}{\Omega}_{k l m n} N^{2} C_{n} C_{k} u_{m}-\stackrel{\circ}{\Lambda}_{m k l} \omega^{2} N^{2} C_{m} C_{k} \varphi, \\
\stackrel{\circ}{k m n}_{k} \omega^{2} N^{2} C_{m} C_{k} u_{n}+\stackrel{\circ}{\varepsilon}_{k n} \omega^{2} N^{2} C_{n} C_{k} \varphi=0 .
\end{gathered}
$$

From which we deduce:

$$
\begin{gathered}
\stackrel{\circ}{\Omega l m n}_{k} N^{2} C_{n} C_{k} u_{m}+\stackrel{\circ}{\Lambda}_{m k l} N^{2} C_{m} C_{k} \varphi-\stackrel{\circ}{\rho} u_{l}=0, \\
\stackrel{\circ}{\Lambda m n}_{k{ }^{2} C_{m} C_{k} u_{n}-\stackrel{\circ}{\varepsilon}_{k n} N^{2} C_{n} C_{k} \varphi=0 .}
\end{gathered}
$$

Thus:

$$
\begin{gathered}
\stackrel{\circ}{\Omega}_{k l m n} C_{n} C_{k} u_{m}+\stackrel{\circ}{\Lambda}_{m k l} C_{m} C_{k} \varphi-\stackrel{\circ}{\rho} \frac{u_{l}}{N^{2}}=0, \\
\stackrel{\circ}{k m n}_{m} C_{m} C_{k} u_{n}-\stackrel{\circ}{\varepsilon}_{k n} C_{n} C_{k} \varphi=0 .
\end{gathered}
$$

Taking $V=\frac{1}{N}$ and $\varphi=u_{4}$ we obtain:

$$
\stackrel{\circ}{\Omega}_{k l m n} C_{n} C_{k} u_{m}+\stackrel{\circ}{\Lambda}_{m k l} C_{m} C_{k} u_{4}-\stackrel{\circ}{\rho} v^{2} u_{l}=0,
$$




$$
\stackrel{\circ}{\Lambda}_{k m n} C_{m} C_{k} u_{n}-\stackrel{\circ}{\varepsilon}_{k n} C_{n} C_{k} u_{4}=0,
$$

which gives:

$$
\left(\begin{array}{cccc}
\stackrel{\circ}{\Gamma}_{11}-\stackrel{\circ}{\rho} V^{2} & \stackrel{\circ}{\Gamma}_{12} & \stackrel{\circ}{\Gamma}_{13} & \stackrel{\circ}{\gamma}_{1} \\
\stackrel{\circ}{\Gamma}_{21} & \stackrel{\circ}{\Gamma}_{22}-\stackrel{\circ}{\rho} V^{2} & \stackrel{\circ}{\Gamma}_{23} & \stackrel{\circ}{\gamma}_{2} \\
\stackrel{\circ}{\Gamma}_{31} & \stackrel{\circ}{\Gamma}_{32} & \stackrel{\circ}{\Gamma}_{33}-\stackrel{\circ}{\rho}^{2} V^{2} & \stackrel{\circ}{\gamma}_{3} \\
\stackrel{\circ}{\gamma}_{1} & \stackrel{\circ}{\gamma}_{2} & \stackrel{\circ}{3}_{3} & -\stackrel{\circ}{\varepsilon}
\end{array}\right)\left(\begin{array}{l}
u_{1} \\
u_{2} \\
u_{3} \\
u_{4}
\end{array}\right)=0
$$

where

$$
\begin{gathered}
\stackrel{\circ}{\Gamma}_{l m}=\stackrel{\circ}{\Omega}_{k l m n} C_{n} C_{k}=c_{k l m n}+\stackrel{\circ}{S}_{k n} \delta_{l m}-e_{k m n} \stackrel{\circ}{E}_{l}-e_{n k l} \stackrel{\circ}{E}_{m}-\eta_{k n} \stackrel{\circ}{E}_{l} \stackrel{\circ}{E}_{m} C_{k} C_{n}, \\
\stackrel{\circ}{\gamma_{l}}=\stackrel{\circ}{\Lambda}_{m k l} C_{m} C_{k}=\left(e_{m k l}+\eta_{m k} \stackrel{\circ}{E}_{l}\right) C_{m} C_{k} \\
\stackrel{\circ}{\varepsilon} \stackrel{\circ}{\varepsilon}_{k n} C_{k} C_{n}=\left(\delta_{k n}+\eta_{k n}\right) C_{k} C_{n} .
\end{gathered}
$$

System (13) represents the propagation condition of the inhomogeneous plane waves inside the previous materials and is equivalent to:

$$
\left(\begin{array}{ll}
\stackrel{\circ}{Q}_{l m} & \stackrel{\circ}{Q}_{l 4} \\
\stackrel{\circ}{Q}_{4 m} & \stackrel{\bigcirc}{44}_{44}
\end{array}\right)\left(\begin{array}{c}
u_{m} \\
u_{4}
\end{array}\right)=0,=\overline{1,3},
$$

where $Q$ is the electroacustic tensor and has the following components:

$$
\begin{gathered}
\stackrel{\circ}{Q m}_{l m}=N^{2} \stackrel{\circ}{\Omega l m n}_{k l} C_{n} C_{k}-\stackrel{\rho}{\rho} \delta_{l m}, \\
\stackrel{\circ}{Q}_{l 4}=N^{2} \stackrel{\circ}{\Lambda m l}_{m} C_{m} C_{k}, \\
\stackrel{\circ}{Q m}_{4 m}=N^{2} \stackrel{\circ}{\Lambda}_{k l m} C_{l} C_{k}, \\
\stackrel{\circ}{Q}_{44}=-N^{2} \stackrel{\circ}{\varepsilon}_{k n} C_{n} C_{k} .
\end{gathered}
$$

Note that the tensor $\mathbf{Q}$ is symmetric for the general anisotropy. 


\section{3 .Inhomogeneous plane waves in isotropic solids subject to initial electro-mechanical fields}

In the particular case of an isotropic material, the elasticity tensor contains two independent components. Using Voight's convention we have:

$$
\left(\begin{array}{cccccc}
c_{11} & c_{12} & c_{12} & 0 & 0 & 0 \\
c_{12} & c_{11} & c_{12} & 0 & 0 & 0 \\
c_{12} & c_{12} & c_{11} & 0 & 0 & 0 \\
0 & 0 & 0 & c_{66} & 0 & 0 \\
0 & 0 & 0 & 0 & c_{66} & 0 \\
0 & 0 & 0 & 0 & 0 & c_{66}
\end{array}\right)
$$

with $c_{11}=\lambda+2 \mu, c_{12}=\lambda$ and $c_{66}=\left(c_{11}-c_{12}\right) / 2=\mu$. Here $\lambda$ and $\mu$ are Lame's coefficients.

The dielectric tensor has only one component, hence

$$
\eta=\left(\begin{array}{lll}
\eta & 0 & 0 \\
0 & \eta & 0 \\
0 & 0 & \eta
\end{array}\right)
$$

and $\varepsilon=1+\eta$.

From (14) now gives:

$$
\begin{gathered}
\stackrel{\circ}{\Gamma}_{11}=\left(\stackrel{\circ}{c}_{11}+\stackrel{\circ}{S}_{11}\right) C_{1}^{2}+\left(\stackrel{\circ}{c}_{66}+\stackrel{\circ}{S}_{22}\right) C_{2}^{2}+\left(\stackrel{\circ}{c}_{66}+\stackrel{\circ}{S}_{33}\right) C_{3}^{2}-\eta\left(C_{1}^{2}+C_{2}^{2}+C_{3}^{2}\right) \stackrel{\circ}{E}_{1}^{2}+(21) \\
+2 \stackrel{\circ}{S}_{12} C_{1} C_{2}++2 \stackrel{\circ}{S}_{13} C_{1} C_{3}+2 \stackrel{\circ}{S}_{23} C_{2} C_{3}, \\
\stackrel{\circ}{\Gamma}_{12}=\stackrel{\circ}{\Gamma}_{21}=-\eta\left(C_{1}^{2}+C_{2}^{2}+C_{3}^{2}\right) \stackrel{\circ}{E}_{1} \stackrel{\circ}{E}_{2}+\left(\stackrel{\circ}{c}_{12}+\stackrel{\circ}{c}_{66}\right) C_{1} C_{2} \\
\stackrel{\circ}{\Gamma}_{13}=\stackrel{\circ}{\Gamma}_{31}=-\eta\left(C_{1}^{2}+C_{2}^{2}+C_{3}^{2}\right) \stackrel{\circ}{E}_{1} \stackrel{\circ}{E}_{3}+\left(\stackrel{\circ}{c}_{12}+\stackrel{\circ}{c}_{66}\right) C_{1} C_{3}, \\
\stackrel{\circ}{\Gamma}_{22}=\left(\stackrel{\circ}{c}_{66}+\stackrel{\circ}{S}_{11}\right) C_{1}^{2}+\left(\stackrel{\circ}{c}_{11}+\stackrel{\circ}{S}_{22}\right) C_{2}^{2}+\left(\stackrel{\circ}{c}_{66}+\stackrel{\circ}{S}_{33}\right) C_{3}^{2}-\eta\left(C_{1}^{2}+C_{2}^{2}+C_{3}^{2}\right) \stackrel{\circ}{E}_{2}^{2}+ \\
+2 \stackrel{\circ}{S}_{12} C_{1} C_{2}+\stackrel{\circ}{S}_{13} C_{1} C_{3}+2 \stackrel{\circ}{S}_{23} C_{2} C_{3}, \\
\stackrel{\circ}{\Gamma}_{23}=\stackrel{\circ}{\Gamma}_{32}=-\eta\left(C_{1}^{2}+C_{2}^{2}+C_{3}^{2}\right) \stackrel{\circ}{E}_{2} \stackrel{\circ}{E}_{3}+\left(\stackrel{\circ}{c}_{12}+\stackrel{\circ}{c}_{66}\right) C_{2} C_{3}, \\
\stackrel{\circ}{\Gamma}_{33}=\left(\stackrel{\circ}{c}_{66}+\stackrel{\circ}{S}_{11}\right) C_{1}^{2}+\left(\stackrel{\circ}{c}_{66}+\stackrel{\circ}{S}_{22}\right) C_{2}^{2}+\left(\stackrel{\circ}{c}_{11}+\stackrel{\circ}{S}_{33}\right) C_{3}^{2}-\eta\left(c_{1}^{2}+c_{2}^{2}+c_{3}^{2}\right) \stackrel{\circ}{E}_{3}^{2}+
\end{gathered}
$$




$$
+2 \stackrel{\circ}{S}_{12} C_{1} C_{2}++2 \stackrel{\circ}{S}_{13} C_{1} C_{3}+2 \stackrel{\circ}{S}_{23} C_{2} C_{3} .
$$

From (15) we also obtain:

$$
\begin{aligned}
& \stackrel{\circ}{\gamma}_{1}=(1+\eta)\left(C_{1}^{2}+C_{2}^{2}+C_{3}^{2}\right) \stackrel{\circ}{E}_{1}, \\
& \stackrel{\circ}{\gamma}_{2}=(1+\eta)\left(C_{1}^{2}+C_{2}^{2}+C_{3}^{2}\right) \stackrel{\circ}{E}_{2}, \\
& \stackrel{\circ}{\gamma}_{3}=(1+\eta)\left(C_{1}^{2}+C_{2}^{2}+C_{3}^{2}\right) \stackrel{\circ}{E}_{3} .
\end{aligned}
$$

and from (16) we obtain:

$$
\stackrel{\circ}{\varepsilon}=(1+\eta)\left(C_{1}^{2}+C_{2}^{2}+C_{3}^{2}\right) .
$$

\section{1 .Direct dyad axis}

In this case, we suppose that $x_{3}$ is a direct dyad axis (this means the plane $0 x_{1} x_{2}$ is normal to a direct axis of order two). We have $A_{2} \| x_{3}$ and we are in the class 2 of the monoclinic system.

If we consider the particular case of isotropic directional bivectors, we may choose $\mathbf{C}=(1, i, 0)$. In this case, the inhomogeneous wave is circularly polarized in a plane normal to the dyad axis $x_{3}$.

If $\stackrel{\circ}{E}_{1}=\stackrel{\circ}{E}_{2}=0$, we obtain $\stackrel{\circ}{\Gamma}_{13}=\stackrel{\circ}{\Gamma}_{23}=0$ si $\stackrel{\circ}{\gamma}_{1}=\stackrel{\circ}{\gamma}_{2}=\stackrel{\circ}{\gamma}_{3}=\stackrel{\circ}{\varepsilon}=0$. The system (17) reduces to two independent subsystems:

a) The first subsytstem:

$$
\left(\begin{array}{cc}
\stackrel{\circ}{\Gamma}_{11}-\stackrel{\circ}{\rho} V^{2} & \stackrel{\circ}{\Gamma}_{12} \\
\stackrel{\circ}{\Gamma}_{12} & \stackrel{\circ}{\Gamma}_{22}-\stackrel{\circ}{\rho} V^{2}
\end{array}\right)\left(\begin{array}{l}
u_{1} \\
u_{2}
\end{array}\right)=0,
$$

where

$$
\begin{gathered}
\stackrel{\circ}{\Gamma}_{11}=\left(\stackrel{\circ}{c}_{11}+\stackrel{\circ}{S}_{11}\right)-\left(\stackrel{\circ}{c}_{66}+\stackrel{\circ}{S}_{22}\right)+2 \stackrel{\circ}{S}_{12} i, \\
\stackrel{\circ}{\Gamma}_{12}=\stackrel{\circ}{\Gamma}_{21}=\left(\stackrel{\circ}{c}_{12}+\stackrel{\circ}{c}_{66}\right) i, \\
\stackrel{\circ}{\Gamma}_{22}=\left(\stackrel{\circ}{c}_{66}+\stackrel{\circ}{S}_{11}\right)-\left(\stackrel{\circ}{c}_{11}+\stackrel{\circ}{S}_{22}\right)+2 \stackrel{\circ}{S}_{12} i .
\end{gathered}
$$

The solution of this subsystem coresponds to $\stackrel{\circ}{P}_{2}$ guided wave. This problem defines a non-piezoelectric guided wave, polarized in the plane $x_{1} x_{2} . \stackrel{\circ}{P}_{2}$ depends on the initial stress field, only.

b) The solution of this subsystem is a single equation, as follows: 


$$
\left(\stackrel{\circ}{\Gamma}_{33}-\stackrel{\circ}{\rho} V^{2}\right) u_{3}=0,
$$

where $\stackrel{\circ}{\Gamma}_{33}=\left(\stackrel{\circ}{S}_{11}-\stackrel{\circ}{S}_{22}\right)+2 i \stackrel{\circ}{S}_{12}$.

The solution of this subsystem is a transverse-horizontal wave, with polarization after the axis $x_{3}$. This wave is piezoelectric, depends on the initial mechanical fields, and is denoted by $\frac{\circ}{T H}$.

\subsection{Inverse dyad axis (mirror plane)}

We now suppose the plane $x_{1} x_{2}$ is normal to an inverse dyad axis $\left(x_{3}\right.$ in our case), which is equivalent to the fact that the plane $x_{1} x_{2}$ is parallel to a mirror plane $M$.It follows that the material belongs to the class $m$ of the monoclinic system $\left(M \perp x_{3}\right)$. In this case, the electroacoustic tensor $Q$ is symmetric with complex components.

If we consider the particular case of isotropic directional bivectors, we may choose $\boldsymbol{C}=(1, i, 0)$. In this case, the inhomogeneous wave is circulary polarized in a normal plane to the inverse dyad axis $x_{3}$.

Moreover, if $\stackrel{\circ}{E}_{3}=0$ we obtain $\stackrel{\circ}{\Gamma}_{13}=\stackrel{\circ}{\Gamma}_{23}=0$ and $\stackrel{\circ}{\gamma}_{1}=\stackrel{\circ}{\gamma}_{2}=\stackrel{\circ}{\gamma}_{3}=\stackrel{\circ}{\varepsilon}=0$. In this case, the system (17) reduces to two independent subsystems:

a) The first subsystem:

$$
\left(\begin{array}{cc}
\stackrel{\circ}{\Gamma}_{11}-\stackrel{\circ}{\rho} V^{2} & \stackrel{\circ}{\Gamma}_{12} \\
\stackrel{\circ}{\Gamma}_{12} & \stackrel{\circ}{\Gamma}_{22}-\stackrel{\circ}{\rho} V^{2}
\end{array}\right)\left(\begin{array}{l}
u_{1} \\
u_{2}
\end{array}\right)=0,
$$

where

$$
\begin{gathered}
\stackrel{\circ}{\Gamma}_{11}=\left(\stackrel{\circ}{c}_{11}+\stackrel{\circ}{S}_{11}\right)-\left(\stackrel{\circ}{c}_{66}+\stackrel{\circ}{S}_{22}\right)+2 \stackrel{\circ}{S}_{12} i, \\
\stackrel{\circ}{\Gamma}_{12}=\stackrel{\circ}{\Gamma}_{21}=\left(\stackrel{\circ}{c}_{12}+\stackrel{\circ}{c}_{66}\right) i, \\
\stackrel{\circ}{\Gamma}_{22}=\left(\stackrel{\circ}{c}_{66}+\stackrel{\circ}{S}_{11}\right)-\left(\stackrel{\circ}{c}_{11}+\stackrel{\circ}{S}_{22}\right)+2 \stackrel{\circ}{S}_{12} i .
\end{gathered}
$$

A solution of this system is an inhomogeneous plane wave, polarized into the plane $x_{1} x_{2}$ and depending on the initial stress fields. It also corresponds to $\stackrel{\circ}{\stackrel{P}{2}_{2}}$ from the problem of guided wave propagation.

b) The second subsystem reduces to a single equation, as follows:

$$
\left(\stackrel{\circ}{\Gamma}_{33}-\stackrel{\circ}{\rho} V^{2}\right) u_{3}=0,
$$

where $\stackrel{\circ}{\Gamma}_{33}=\left(\stackrel{\circ}{S}_{11}-\stackrel{\circ}{S}_{22}\right)+2 i \stackrel{\circ}{12}_{12}$.

Its root is linked to a transverse-horizontal wave, with polarization after the axis $x_{3}$, non-piezoelectric and influenced by the initial stress field, only. It corresponds to $T H$ from the problem of guided wave propagation. 


\section{Conclusions}

In this paper, we obtained the condition of inhomogeneous plane wave propagation in isotropic solid crystals subject to initial electromechanical fields. For particular isotropic directional bivectors we derive the decomposition of the propagation condition, and we show that the specific coefficients are similar

to the case of guided waves propagation in isotropic solid crystals subject to a bias.

\section{References}

[1] Ph. Boulanger, M. Destrade, M. Hayes, Inhomogeneous "longitudinal" circularly-polarized plane waves in anisotropic elastic crystals, Acta Acustica, 92(2006), 247-250.

[2] Ph. Boulanger, M. Hayes, Electromagnetic plane waves in anisotropic media an approach using bivectors,Phil. Trans. R. Soc. A, 330(1990), 335393.

[3] Ph. Boulanger, M. Hayes, Bivectors and waves in Mechanics and Optics Chapman \& Hall, New Delhi, 1993.

[4] S. Chirita, I. Ghiba, Inhomogeneous plane waves in elastic materials with voids, Wave Motion, 47(2010), 333-342.

[5] J.W. Gibbs, Elements of vector analysis, Scientific papers vol.2, part 2, Doves Publication, New York, 1961.

[6] W.R. Hamilton, Lectures on quaternions, Hodges and Smith, Dublin, 1853.

[7] M. Hayes, Inhomogeneous plane waves, Arch. Rational Mech. Anal., 85(1984), 41-79.

[8] D. Royer, E. Dieulesaint, Elastic waves in solids, vol. I, Springer, Berlin, 2000.

[9] A.C. Eringen, G.A. Maugin, Electrodynamics of continua, Springer, New York, 1990.

[10] O. Simionescu-Panait, The influence of initial fields on wave propagation in piezoelectric crystals, Int. J. of Appl. Electromagnetics and Mech., 241$252,2000$. 
[11] O. Simionescu-Panait, Wave propagation in cubic crystals subject to initial mechanical and electric fields, ZAMP, 53, 1038-1054, 2002.

[12] O. Simionescu-Panait, Waves in strained polarized media, Special topics in the theory of piezoelectricity,(Ed. J.Yang) , 169-246, Springer, New York, 2009.

[13] O. Simionescu-Panait, Propagation of attenuated waves in isotropic solids subject to initial electro-mechanical fields, Proc. Internat. Conf. NewTrends in Continuum Mechanics, 267-275, Ed. Theta, Bucharest, 2005.

[14] O. Simionescu-Panait, Initial fields impact on attenuated wave propagation in isotropic solids, Math. Reports 8(58), 2(2006), 239-250.

[15] O. Simionescu-Panait, Geometry of electroelastic materials subject to biasing fields, Balkan Journal of Geometry and Its Applications, 14(2009), 91-101.

[16] O. Simionescu-Panait, Inhomogeneous plane waves in monoclinic crystals subject to a bias, PAMM, 11(2011), 637-638.

[17] O. Simionescu-Panait, TH-waves propagation in crystals subject to initial fields,Bulletin of the Transilvania University of Brasov, vol 5(54) 2012, 259-274.

[18] J.L. Synge, The Petrov classification of gravitation hields, Commun Dublin Inst. Adv. Stud, A,15/1960.

Livia Harabagiu

Department of Mathematics,University of Bucharest,

Bucharest,Romania

harabagiu_livia@yahoo.com 